Max-Planck-Institut für demografische Forschung

Max Planck Institute for Demographic Research

Doberaner Strasse $114 \cdot$ D-18057 Rostock $\cdot$ GERMANY

Tel +49 (0) 3812081 - 0; Fax +49 (0) 3812081 - 202;

http://www.demogr.mpg.de

MPIDR WORKING PAPER WP 2002-007

FEBRUARY 2002

\title{
Diffusion Processes \\ and \\ Event History Analysis
}

Norman Braun (Norman.Braun@soziologie.uni-muenchen.de)

Henriette Engelhardt (engelhardt@demogr.mpg.de)

This working paper has been approved for release by: Jan M. Hoem (hoem@demogr.mpg.de)

Head of the Laboratory of Contemporary European Fertility and Family Dynamics.

(C) Copyright is held by the authors.

Working papers of the Max Planck Institute for Demographic Research receive only limited review. Views or opinions expressed in working papers are attributable to the authors and do not necessarily reflect those of the Institute. 


\title{
Diffusion Processes and Event History Analysis ${ }^{1}$
}

\author{
Norman Braun \\ University of Munich, Institute for Sociology
}

Henriette Engelhardt

Max Planck Institute for Demographic Research, Rostock

February 8, 2002

\footnotetext{
${ }^{1}$ We wish to thank Josef Brüderl for detailed comments and Andreas Diekmann for discussions.
} 


\title{
Diffusion Processes and Event History Analysis
}

\begin{abstract}
Several authors (e.g., Brüderl, Diekmann, Yamaguchi) derive hazard rate models of event history analysis from social diffusion processes. This paper also focuses on the integration of diffusion research and survival analysis. After a discussion of Diekmann's flexible diffusion model, we present an alternative approach which clarifies theoretical differences between popular rate models (e.g., the exponential model, log-logistic model, sickle model). Specifically, this approach provides a new rationale for the generalized log-logistic model in the sense of a flexible infection process. In cases with bell-shaped duration dependence, it thus allows a test for social contagion as a result of random contacts between actual and potential adopters. An application to divorce data serves as an illustration.
\end{abstract}

\section{Introduction}

Techniques of event history modeling are finding increasing use in the social sciences. The range of applications includes labor market studies, demographic analyses, mobility studies, studies in organizational ecology, political science, and so on. Undoubtedly, the availibility of panel and retrospective data sets (e.g., the German Socioeconomic Panel, Family and Fertility Surveys), different introductory textbooks (e.g., Blossfeld and Rohwer 1995; Courgeau and Lelièvre 1992; Diekmann and Mitter 1984; Lancester 1996) as well as suitable software (e.g., Rohwers TDA) has promoted the spreading of these methods.

Parametric approaches to survival analysis have, however, at least one deficiency: a theoretical explanation for the estimated statistical model is usually missing. Generally, for any given event data the shape of the empirical transition rate is first deterimined by using non-parametric procedures (e.g., the Kaplan Meier estimator). This empirical evidence about the course of the so-called hazard rate or risk function is then used for the selection of a suitable parametric model, which is then estimated above all by the maximum likelihood method, taking account of covariates. Theoretical considerations rarely refer to the selected hazard rate model, but rather to the selection and interpretation of covariates for the explanation of the risk process by endogenization of at least one process parameter. This is to a large extent unproblematic if the shape of the empirical risk function suggests a unique parametric model for the statistical estimation. 
However, many social processes are characterized by similarly running rates, which can be described (from a theoretical point of view) by different models. Such a situation results for instance in the case of an approximately bell-shaped hazard rate. Such a form is found more or less regularly in the investigation of marriage behavior, divorce risk or mobility in firms. For its representation different non-monotonous hazard rate models can be used. In addition to the gamma model, the log-normal model, or the log-logistic standard model Diekmann and Mitter's (1983, 1984a) sickle model and Brüderl and Diekmann's (1995) generalized log-logistic model can be applied to the data. A theoretically justified selection between such alternative models would be desirable.

In order to obtain such a such theoretical foundation, some authors (e.g., Brüderl and Diekmann 1995; Diekmann 1990, 1992; Yamaguchi 1994) link event history models with models from diffusion research (e.g., Hamblin, Jacobsen and Miller 1973; Mahajan and Peterson 1985; Rogers 1983). Here, the idea that the respective event history model model can be interpreted in terms of a social diffusion process is fundamental. The common starting point of these works is in each case a general differential equation which covers a multiplicity of diffusion hypotheses as special cases and so supplies a process-theoretical rationale for different hazard rate models. Similarly shaped rates may also correspond to quite different diffusion processes, so that the knowledge of the underlying process hypotheses can in principle be helpful in the selection of a suitable rate model. Under similar conditions (e.g., goodness of fit) preference should be given to that event history model which is accompanied by a theoretically more plausible diffusion hypothesis for the application under consideration. If, on the other hand, there exists a unique hazard rate model that is clearly preferable from a statistical point of view, then the knowledge of the compatible diffusion process at least promotes an understanding of the process under consideration.

The connection between diffusion research and event history analysis is the focus of the present paper. After an introduction of central concepts we discuss Diekmann's (1990, 1992) flexible diffusion model (Section 2). Afterwards, we present a more restrictive model (Section 3). This approach illustrates differences in the underlying theoretical processes between established event history models (e.g., the exponential model, log-logistic model, sickle model). Specifically, this approach provides a new rationale for Brüderl and Diekmann's (1995) generalized log-logistic model in the sense of a flexible infection process. In cases with bell-shaped duration dependence, it thus allows a test for social contagion due to random contacts between actual and potential adopters. An application to divorce data serves as an illustration (Section 4). Especially because it is unlikely that divorce has much to do with social contagion, the suggested approach of connecting diffusion research and event history analysis can be tested to see if it leads to plausible results. 


\section{Foundations}

\subsection{Concepts}

Under consideration is a social system with a large number of individuals who, in a given period of time, are confronted with a binary and absorbing event, whose occurrence can be interpreted as result of a decision (e.g., marriage, divorce, adopting new technologies). ${ }^{2}$ Let $T$ be a nonnegative continuous random variable representing the duration until the occurrence of an event (arrival time or waiting time, depending upon the viewpoint) with distribution function $F(t)$ and density function $f(t)=d F(t) / d t$. Because $F(t)$ defines the proportion of the population which had already experienced the event up to time $t, 1-F(t)$ represents the complementary proportion without the event up to this point in time. The hazard rate $h(t)=f(t) /(1-F(t))$ then gives (approximately) the conditioned probability of a change in status in the (very small) time interval $[t, t+\Delta t]$, if the event has still not occurred before $t$.

These concepts were originally introduced and interpreted in the sense of event history analysis. From the perspective of diffusion research, other terms can be used which are at least partly borrowed from epidemiology (cf., e.g., Kramer 1988). Since $F(t)$ defines the proportion of prior adopters or those "infected" at time $t$, this quantity indicates the period-specific prevalence rate. The derivative or density $d F(t) / d t=f(t)$ can thus be interpreted as the increase in prevalence during a (short) additional time interval. ${ }^{3}$ Because $1-F(t)$ defines the respective proportion of potential adopters (population at risk) and $h(t)=f(t) /(1-F(t))$ applies by definition, the hazard rate $h(t)$ can be interpreted as the "incidence rate". ${ }^{4}$ It captures the (relative) influx to the group of adopters during the brief time interval $[t, t+\Delta t]$.

\footnotetext{
${ }^{2}$ Biologically or technically caused events are thereby impossible, such as deaths or damage to a machine, for instance.

${ }^{3}$ Because $d F(t) / d t=f(t)$ expresses the increase in the proportion of adopters per time unit, $f(t) / F(t)$ indicates the respective growth rate of the prevalence.

${ }^{4}$ According to Kramer (1988: 27-32) the prevalence rate defines the proportion of adopters in a certain population at a fixed point in time. In contrast, the incidence rate always refers to a time interval. It defines the proportion of non-adopters from this population who become adopters during a certain period. In our context (ignoring absolute sizes) the incidence rate corresponds therefore to the hazard rate $h(t)$, while the respective prevalence rate is given by the distribution function $F(t)$. For a very small prevalence rate of a feature (e.g., the proportion of regular consumers of hard drugs in the total population) one can capture the incidence rate by the density function $f(t)$, because in this case $f(t)$ and $h(t)=f(t) /(1-F(t))$ are approximately equal.
} 
Thus, a process of the spreading of behaviors (or the occurrence of events) takes account of a connection between incidence and prevalence. In other words, a diffusion process reflects a functional relationship between $h(t)$ and $F(t)$. Due to the large number of possible relations and the definition of the hazard rate, the density $f(t)$ should be expressed as a sufficiently general function of the prevalence $F(t)$. We now turn to such an integration of diffusion research and event history analysis.

\subsection{Flexible Diffusion Model}

A general model for connecting the logic of diffusion and event history analysis is suggested by Diekmann (1990, 1992). His model is based on differential equations. Accordingly, the spread of the feature under consideration or the occurrence of the relevant event can be thought of as a consequence of the information flow within the system. The adoption of a behavior or the occurrence of the event are a result of information that is passed on via interaction with adopters or by sources of influence (e.g., the mass media) that are present throughout the system.

To capture different diffusion processes Diekmann makes the assumption that the information transfer takes place by contacts between a subset of the already infected and not infected portions of the population. ${ }^{5}$ The mixing assumption is $p(t)=F(t)^{m}(1-$ $F(t))^{n}$, where $m$ and $n$ are arbitrarily selectable parameters not interpreted as to content. Because this product indicates the proportion of interactions between sections of the population at $t$, one can view $p=p(t)$ as the probability of an informative and thus potentially infectious contact for members of the risk population at time $t$. Its weighting with an arbitrarily selectable adoption rate $s(t)>0$ determines then the increase on the proportion of the adopters per unit of time:

$$
\frac{d F(t)}{d t}=s(t) F(t)^{m}(1-F(t))^{n}
$$

Therefore, the corresponding hazard rate is:

\footnotetext{
${ }^{5}$ The related assumption of a "homogeneous mixing" of the population concerning infection status characterizes standard models in epidemiology as well (e.g., Anderson and May 1991; Bailey 1975). The actors do not differ regarding age, education, sex etc. Research on socially structured diffusion expanded enormously as a result of the AIDS epidemic. Morris (1994) presents an overview and a modeling suggestion for dealing with non-homogeneous mixing in epidemiological models which is based on loglinear methods. Strang (1991) discusses the role of event history analysis when introducing socialstructural features into models of social diffusion.
} 


$$
h(t)=s(t) F(t)^{m}(1-F(t))^{n-1},
$$

such that very different connections between incidence and prevalence can be illustrated. ${ }^{6}$ The type of diffusion process as well as the interpretation of the adoption rate $s(t)$ is determined to a large degree by the selection of the parameters $m$ and $n$. In particular, three process types can be differentiated:

- The parameter combination $m=1$ and $n=1$ defines the probability of a potential infection of potential adopters per unit of time through $p(t)=F(t)(1-F(t))$. Individual instances of adopting behavior or of events can thus be interpreted as resulting from coincidental interactions between prior and potential adopters. This scenario is based on the assumption of homogeneous mixing after contagion. Thus, this illustrates pure infection processes. According to Diekmann $s(t)$ represents the contagion rate for interactions between actors with and without a given feature (event). ${ }^{7}$

- As a result of the parameter constellation $m=0$ and $n=1$, the probability of a potentially influential contact for potential adopters per unit of time arises through $p(t)=1-F(t)$, the proportion of the population at risk. Adopting behavior can be understood here as a result of contacts of this part of the population with the total system. One can thus regard them as consequences of the influence of system-wide sources (e.g., mass media), so that $s(t)$ is interpretable in Diekmanns sense as a timedependent influence rate. ${ }^{8}$

- The parameter combination $m=0$ and $n=2$ determines the probability of a potentially influential contact for members of the risk population per unit of time through $p(t)=(1-F(t))^{2}$. In this scenario interactions between potential adopters play the crucial role. Diekmann (1990) speaks in this case of matching processes (e.g., a sales contract, marriage), in which $s(t)$ is to be understood as the timedependent matching rate.

Certainly, Diekmanns approach offers an elegant connection between diffusiontheoretical logic and event-analytic methodology. However there are also arguments that

\footnotetext{
6 Diekmann (1990, 1992), Yamaguchi (1994), and Brüderl and Diekmann (1995) present detailed discussions of special cases of this approach for integrating diffusion research and event history analysis.

7 The example of such a infection process that is probably the most well-known is the logistic model applied by Coleman, Katz and Menzel (1957), which results from (1) for $m=n=1$ and $s(t)=\alpha$.

${ }^{8}$ The classical example of such an influence process is the exponential model, which was already applied by Coleman, Katz and Menzel (1957). Instead of "influence by the system", one speaks frequently of "external influence" in the diffusion-theoretical literature (e.g., Mahajan and Peterson 1985), whereas
} 
justify the formulation of an alternative approach for the integration of diffusion research and event history analysis:

- First, the unlimitedness of the two parameters $m$ and $n$ is striking. According to the logic of the diffusion approach one can interpret $p(t)=F(t)^{m}(1-F(t))^{n}$ as the probability of an informative contact for potential adopters. Because $F(\cdot)$ is a distribution function, however, $p(t)=F(t)^{m}(1-F(t))^{n} \leq 1$ must apply. This condition is possibly violated with unrestricted values of $m$ and $n$ (example: $m=-1, n=1$ and $F=0.4)$. It is fulfilled, for example, by the restrictions $m \geq 0$ and $n \geq 0$.

- If one is limited to the scenario $m \geq 0$ and $n \geq 0$, then $m$ and $n$ determine the interacting parts of the groups of the prior and potential adopters. It is unclear whether and how the two parameters $m$ and $n$ can be interpreted contentwise. It is not clear here for instance why $m=n=0$ always means that $p=1$, but $m \rightarrow \infty$ and/or $n$ $\rightarrow \infty$ always leads to $p=0$.

- Apart from the selection of the respective interaction pattern by specification of $m$ and $n$, the approach also permits an arbitrary definition of the adoption function $s(t)$. In principle the number of parameters and the functional form of $s(t)$ can be freely determined. This flexibility is reflected in the fact that there are different diffusiontheoretical explanations for one and the same hazard rate model. Thus, the loglogistic standard model can be interpreted both as an infection process and a matching process. ${ }^{9}$ For other standard models of event history analysis (e.g., exponential distribution, generalized log-logistic model, sickle model) ambiguous diffusion-theoretical interpretations are also possible since one can freely choose $m, n$ and $s(t)$ (Braun 1998). In order to arrive at a unique derivative of individual event models from (1), one could follow, e.g., Yamaguchi (1994) and concentrate on pure contagion processes $(m=n=1)$ with flexible selection of the adoption rate $s(t)$.

In the following section we will focus on an alternative way to avoid such ambiguities and interpretation problems. This approach deviates substantially from Diekmanns approach in at least two aspects. The arbitrary adoption function $s(t)$ is replaced by a flexible function, and the parameters $m$ and $n$, which are unclear as far as content is concerned, are eliminated in favor of an interpretable quantity. Although fundamental

infection stemming from contacts between actors with and without the feature is referred to as "internal influence".

9 According to Diekmann (1992) one gets the infection-theoretical explanation for the log-logistic standard model by the parameter selection $m=n=1$ and the infection rate $\mathrm{s}(\mathrm{t})=s(t)=\alpha / t$ in (1), whereas the interpretation of the model as a matching process results from the specifications $m=0, n=2$ and $s(t)$ $=\alpha \gamma(\not t)^{\alpha-1}$ with $\alpha, \gamma>0$ in (1). 
considerations and established concepts are maintained wherever possible, these differences already find expression in the model assumptions we specify below.

\section{An Alternative Model}

\subsection{Model Assumptions}

Once again we assume that spreading processes result from appropriate information about the characteristic or event under consideration and the respective willingness of the actors to adopt them. Sources of information are interactions between actors in the system. However, only a subset of all contacts is informative as regards the feature and not every informative contact necessarily leads to the occurrence of an event. It therefore makes sense to determine first the probability of an interaction with an adopter per unit of time and then to specify accordingly the time-dependent adoption function of potential adopters. A simple assumption for linking these quantities results finally in a flexible hazard rate function and thus in a general diffusion model.

\section{Probability of Informative Contacts}

The quantities $F(t)$ and $1-F(t)$ represent the respective proportions of prior and potential adopters in the system. If all actors (independent of their status concerning the event) possess the same chance of beginning an interaction, $F(t)$ determines probability of an individual coincidentally meeting a prior adopter. On the other hand, $(1-F(t))$ determines the probability that such a contact is not with an adopter and therefore uninformative concerning the event. If one supposes now for each actor in the system $k$ statistically independent contacts per unit of time, then $(1-F(t))^{k}$ gives the probability that an individual has had no contact with an adopter. Thus,

$$
w(t)=1-(1-F(t))^{k}
$$

is the probability that at least one of $k$ statistically independent contacts of any given actor with an adopter will occur and is therefore informative concerning the event. ${ }^{10}$ The

\footnotetext{
10 The quantity $w(t)$ defines the probability of an informative contact for each actor in the system, independently of his status concerning the feature. Thus, it differs conceptionally from $p(t)$, the probability of an informative contact for members of the risk population in Diekmanns approach.
} 
function $w(t)$ increases ceteris paribus with $k$, the statistically independent contacts of each actor in the system per time unit: for $k=0, w(t)=0$; for $k=1, w(t)=F(t)$; for $k=$ $2, w(t)=F(t)(2-F(t))$; and $k \rightarrow \infty$ corresponds to $w(t)=1$. Under otherwise identical conditions, $w(t)$ increases along with the prevalence rate $F(t)$. Because the latter usually rises with time, the probability of a potentially influential contact also tends to rise with time passed (since the beginning of the process). A positive or negative time dependency often also characterizes the willingness of potential adopters to adopt the characteristic in question.

\section{The Adoption Function}

In order to achieve a complete parameterization, a sufficiently general adoption function $r(t)>0$ is introduced, which determines the adoption rate per unit of time. ${ }^{11}$ This function possesses the real-number parameters $c>0, \delta$ and $\varepsilon \geq 0:{ }^{12}$

$$
r(t)=c e^{\delta t} t^{\varepsilon-1},
$$

where $e$ defines the base of the natural logarithm and the scaling parameter $c$ can be reparameterized if necessary. ${ }^{13}$ Thus, the adoption rate for $\delta=0$ and $\varepsilon=1$ is a constant. In all other cases it depends on the time $t$, though the effect is influenced substantially by the combination of the respective parameters $\delta$ and $\varepsilon$. The elasticity function of $r(t)$ gives a brief summary of all possibilities. The elasticity of the adoption rate is a linear function of the time passed since the beginning of the process:

$$
\frac{d r(t)}{d t} \frac{t}{r(t)}=(\varepsilon-1)+\delta t
$$

An $1 \%$ increase in the time passed changes the adoption rate by approximately 100 ( $\varepsilon-$ $1+\delta \mathrm{t}) \%$. In the definition of diffusion processes and the corresponding hazard models assumptions about the time elasticity of the willingness of adoption are thereby

\footnotetext{
${ }^{11}$ The flexible function $r(t)$ plays a role similar to that of the arbitrary function $s(t)$ in Diekmanns approach.

${ }^{12}$ The function $r(t)$ is elementarily integrable only for real numbers of $e$. It is a generalization of the gamma density $g=\left(b(b t)^{\alpha-1} e^{-b t}\right) / \Gamma(a)$, in which $a$ and $b$ are positive parameters and $\Gamma(\cdot)$ represents the gamma function. Namely, $r(t)=g(t)$ for $\varepsilon=a>0, \delta=-b<0$ and $c=b^{a} / \Gamma(a)$. Due to the characteristics of the gamma function, $c=b^{a} /(\varepsilon-1)$ ! if $\varepsilon=a$ is a positive real number.

${ }^{13}$ Typical re-parameterizations are $c=\alpha \beta / \gamma$ and $c=\alpha \gamma^{\alpha}$, whereby $\alpha, \beta, \gamma$ represent positive parameters.
} 
formulated. Before this can be made clearer, we first need to introduce an assumption about the determinants of the hazard rate.

\section{Hazard Rate}

The hazard rate or risk function $h(t)$ indicates the conditional probability of a status change for 'feature-free' actors. If one assumes that a feature spreads as a result of contacts between actors in the system, it is plausible that the risk of the occurrence of the event will depend on the probability of an informative contact $w(t)$ and the adoption rate $r(t)$. A high risk of occurrence might exist, in particular, when both the probability of an interaction with an adopter and the willingness of adoption are high. Thus, the following assumption for determinants of the hazard seems to makes sense:

$$
h(t)=r(t) w(t) .
$$

The hazard rate arises then as a result of the product of the time-dependent adoption rate and the probability of a contact which is informative and therefore potentially consequential as regards the event or feature. We have now formulated a general diffusion model on the basis of the postulates for $r(t)$ and $w(t)$ discussed above.

\subsection{Model Conclusions}

The assumptions (3), (4) and (5) determine a flexible risk function which itself implies a general diffusion equation. The model prerequisites result in the hazard function

$$
h(t)=c e^{\delta t} t^{\varepsilon-1}\left(1-(1-F(t))^{k}\right) .
$$

Before the corresponding density function $f(t)$ - and thereby the general diffusion equation - it makes sense to discuss briefly the process-theoretical implications of (7). Because $h(t)=r(t) w(t)$, and $w(t)$ rises with the number of the statistically independent contacts $k, h(t)$ increases with $k$, assuming otherwise identical conditions. If one assumes $0<F(\cdot)<1$, then diffusion processes can be differentiated according to the potential of influence of interactions: 
Contact-dependent spreading: If $k$ is not very large, then $w(t)<1$ for the probability of an informative contact. Thus, apart from the adoption function $r(t)$, this probability influences the hazard rate $h(t)$ and interactions therefore play a rather important role for the spreading process.

Contact-independent spreading: In the case of innumerable contacts per unit of time, we have the opposite result. In the borderline case $k \rightarrow \infty, w(t)=1$, so that the risk function $h(t)$ is determined exclusively by the adoption function $r(t)$. In this scenario the occurrence of an event is independent of the probability of an informative contact, which means that interactions between the system actors play no role in the spreading process.

The distinction between contact-dependent and contact-independent diffusion is of course also relevant for the density $f(t)=d F / d t$ from (7). This results from combining the definition $h(t)=(d F / d t) /(1-F(t))$ with the hazard function (7):

$$
\frac{d F}{d t}=c e^{\delta t} t^{\varepsilon-1}\left(1-(1-F(t))^{k}\right)(1-F(t))
$$

Like Diekmann's differential equation this diffusion equation is explicitly solvable only for certain parameter combinations. TABLE 1 presents a selection according to the diffusion-theoretical interpretation of these special cases: for $k=1$ infection processes are present; for $k=\gamma / \beta$ a flexible model arises in the sense of a generalized contagion process; and for $k \rightarrow \infty$ we have contact-independent propagation processes. In addition to a brief look at the exponential model and the logistic model, we will take a closer look at the remaining special cases from TABLE 1 in the following. Because the latter either exhibit or at least permit a bell-shaped hazard rate curve, this discussion serves also in preparation for the application.

[Table 1 about here]

\section{Exponential Distribution}

One of the process types in TABLE 1 is based on the assumption of innumerable interactions between system actors $(k \rightarrow \infty)$. In this scenario there is always an informative contact concerning the feature per unit of time, so the spreading process 
occurs independently of the interaction pattern. Thus, the diffusion process does not take place via contagion as a result by interactions between potential and prior adopters. Rather, it is driven exclusively by the adoption function.

In TABLE 1 this type of process is represented by, among others, the exponential model, which frequently serves as a reference model in event history analysis. Based on (8), this classical model of a contact-independent spreading process results from assuming, in addition, a constant adoption rate (cf., e.g., Coleman, Katz and Menzel 1957). Assuming $c>0, \delta=0$ and $\varepsilon=1$ :

$$
\frac{d F}{d t}=c(1-F(t)), F(t)=1-e^{-c t}, h(t)=c,
$$

where the integration of the density $f(t)=d F / d t$ yields the distribution function $F(t)$, so that the corresponding hazard rate $h(t)=f(t) /(1-F(t))$ is determined by the constant $c$.

\section{Sickle Models}

In addition to the exponential model, TABLE 1 contains a further model for a spreading process which does not rely on contagion by interactions between prior and potential adopters. The constellation of the contact-independent spreading also characterizes the sickle-shaped hazard function of Diekmann and Mitter (1983, 1984a). Using the positive parameters $c$ and $\lambda$ the "defective" distribution function $F(t)$ and the hazard rate $h(t)$ of the sickle model are given by

$$
F(t)=1-e^{-\lambda c\left(\lambda-(t+\lambda) e^{-t / \lambda}\right)}, h(t)=c t e^{-t / \lambda},
$$

so that in the long run "immunity" is present in the sense of $F(\infty)<1$. The estimated value of $\lambda$ thereby determines the point in time of the highest transition risk (i.e., the maximum of the rate function with $t_{m}=\lambda$ ). Apart from $k \rightarrow \infty$ a diffusion-theoretical explanation for the sickle hypotheses in the sense of (8) requires the parameter specification $c>0, \delta=-1 / \lambda$ and $\varepsilon=2$ :

$$
\frac{d F}{d t}=c t e^{-t / \lambda}(1-F(t)) .
$$


If one views the sickle model on this basis, then two consequences result. On the one hand, innumerable interactions per unit of time guarantee that only the adoption rate plays a role in the spreading of the feature, i.e., the adoption function $r(t)$ corresponds to the hazard function $h(t)$. On the other hand, the transition risk exhibits a linearly decreasing elasticity of time $(d r / d t)(t / r(t))=1-(1 / \lambda) t$. A $1-\%$ increase in time (passed since the beginning of the process) results up to a certain point in time (at $t_{m}=\lambda$ ) in a proportional increase in the willingness of adoption, thereafter, however, in a proportional decrease. After an initial acceleration, the adoption tendency is thus retarded once again, though contacts do not play a role in the spreading process. This contrasts with the logic of infection.

\section{Logistic Models}

Examples of social contagion processes are easy to find. The spreading of certain modes, the acquisition of new technologies, or also the propagation of rumors can always be understood as consequences of informative interactions. Assuming the validity of (8), infection processes result if one supposes a statistically independent contact per unit of time $(k=1)$, which means that the proportion of adopters determines the probability of a given informative contact.

The logistic model indicated in TABLE 1 offers the classical description of infectionlike diffusion processes (e.g., Coleman, Katz and Menzel 1957). It results from (8), if one assumes in addition a constant adoption rate and thus $c>0, \delta=0$ and $\varepsilon=1$ :

$$
\frac{d F}{d t}=c F(t)(1-F(t)), F(t)=\frac{F(0) e^{c t}}{1-\left(1-e^{c t}\right) F(0)}, h(t)=c F(t)
$$

With $F(0)$ we assume a positive initial value of the S-shaped distribution function $F(\cdot)$. Because the adoption rate is determined by the constant $c$, the hazard function $h(t)$ represents only a parallel shift in the distribution function. Thus, the hazard rate of the logistic model also indicates an S-shaped process, i.e., the risk of adoption rises with the process duration. However, there are also infection processes that are accompanied by an entirely different course of risk. 


\section{Log-Logistic Models}

The age-dependent taking up of criminal activities as a result of "bad" contacts or the beginning of regular drug consumption due to interactions with friends who already take drugs could be contact-dependent spreading processes with bell-shaped hazard functions. If one concentrates first on pure infection processes $(k=1)$ and makes the corollary assumption that the adoption function $r(t)=c / t$ is constantly decreasing, then the log-logistic standard model proves to be an example of a pure contagion process, which can accompany with a bell-shaped hazard function.

As Brüderl and Diekmann (1995) show, the log-logistic model can be generalized by introducing an additional parameter $\beta$. If we use the positive parameters $\alpha, \beta$ and $\gamma$ the distribution function $F(t)$ and the hazard function $h(t)$ of the generalized log-logistic model are:

$$
F(t)=1-\left(1+(\not t)^{\alpha}\right)^{-\beta / \gamma}, h(t)=\frac{\alpha \beta(\not t)^{\alpha-1}}{1+(\not t)^{\alpha}},
$$

which results in the log-logistic standard model for $\beta=\gamma>0$. For $\alpha>1$ we have a bellshaped curve for $h(t)$, while $\alpha \leq 1 \mathrm{~h}(\mathrm{t})$ implies a constantly decreasing hazard rate.

Because the generalized log-logistic model contains the log-logistic standard model as a special case and the latter is infection-theoretically explainable, the generalized model should be interpretable as a generalized contagion process. In fact, (8) does permit this interpretation of the generalized log-logistic model. If one combines the assumption $k=\gamma / \beta>0$ in (8) with the specifications $c=\alpha \beta / \gamma>0, \delta=0$, and $\varepsilon=0$, then the density of the generalized log-logistic model can be written as a generalized infection process:

$$
\frac{d F}{d t}=\frac{\beta}{\gamma} \frac{\alpha}{t}\left(1-F(t)-(1-F(t))^{\gamma / \beta}+F(t)(1-F(t))^{\gamma / \beta}\right) .
$$

Thus, the generalized log-logistic model illustrates a further type of process in TABLE 1 - as a flexible model of a contact-dependent diffusion, it is situated between pure infection processes and contact-independent spreading processes. Thus, the estimation results for the generalized log-logistic model facilitate the choice between bell-shaped hazard rate models that are based on different process-theoretical foundations. In particular, they make it possible for us to test the infection hypothesis, which justifies the $\log$-logistic standard model: The sooner $\beta=\gamma$ is fulfilled, the more appropriate is an 
interpretation of a bell-shaped risk process in the sense of a pure contagion process. This reflects the fact that the infection-theoretical interpretation of the log-logistic model for $k=1$ results directly from (14), whereby a constant time elasticity of -1 is assumed for the adoption rate $r(t)$. In addition to the possible contagion-theoretical interpretation, it is thus characteristic for log-logistic models that a $1 \%$ increase in time (passed since beginning of the process) leads to a $1 \%$ reduction in adoption willingness.

These process-theoretical differences between the log-logistic models and the similarly shaped sickle model serve to help in the selection of the suitable statistical model. We will now show this with an investigation of duration data concerning "divorce".

\section{An Illustration: Divorces}

One can hardly view divorces as being the consequence of social contagion processes. An analysis of divorce data can thus show whether our suggested approach of connecting diffusion research and event history analysis leads to plausible results. Our starting point is the well-known fact that divorce data can be adequately described by a bell-shaped risk or hazard rate function (cf., e.g., Diekmann and Engelhardt 1999; Brüderl, Diekmann and Engelhardt 1999). In the following we take a look at the sickle model and the generalized log-logistic model, two parametric models that can illustrate such a shape for the transition rate, although they are based on different processtheoretical considerations. Although we orient ourselves throughout to the traditional procedure for the employment of event-analytic methods in divorce research, the substantial findings of the data analysis are neglected to a large extent. ${ }^{14}$ Our objective is to prove that the process-theoretical considerations discussed above can be of use when choosing between competitive hazard rate models. After a brief description of the data and covariates we will also deal briefly with the statistical procedures that are usually employed in model selection.

\subsection{Data and Variables}

This study is based on the West German Family Survey from 1988, which was administered by the German Youth Institute (DJI). The DJI study is a random sample of

\footnotetext{
${ }^{14}$ Detailed interpretations of the content of similar estimation results can be found in Brüderl, Diekmann and Engelhardt (1997) as well as in Diekmann and Engelhardt (1999).
} 
the entire West German residential population between the ages of 18 and 55 living in private households. A total of 10,043 people participated in a personal interview in which detailed information on their partnership history was collected. Although the response rate was relatively low (52\%), comparisons of the distributions of the sociodemographic variables with official statistics show that deviations are no greater than in other national surveys. Women, married persons, and persons not in the labor force are somewhat oversampled, while family and household type both correspond well with official statistics (Bender et al. 1996). The data are available under code ZA-Nr. 2245 from the Zentralarchiv in Cologne. For the following analyses, we consider only first marriages. There are 7,200 first marriages in the data, of which $12.6 \%$ had ended in divorce at the time of inquiry.

Apart from the central variable "marriage duration" the data set allows us to control for numerous characteristics of the respondents and the couples that are considered to be central divorce determinants (cf., e.g., Engelhardt 2000). These characteristics can thus be included in the analysis as covariables. In detail, the variables are constructed as follows:

- The key variable "marriage duration" is measured in years until a legal divorce or length of marriage until the date of the interview.

- Three dummy variables were constituted for the 1961-70, 1971-80, and 1981-88 "marriage cohorts." The reference category is the 1949-60 marriage cohort.

- The denomination of marriage partners is coded with three dummies: both spouses Catholic ("catholic couple"), neither partner with religious affiliation ("without denomination"), couple with mixed denominations ("mixed denomination"). The reference category embraces couples where both partners have the same denomination (Protestant and other churches).

- If the couple is of mixed nationality, a dummy variable ("partner is foreigner") is coded 1 , otherwise it is 0 .

- If a couple founded a joint household before entering marriage, the dummy variable "premarital cohabitation" equals 1, otherwise it is 0.21 .9 percent of our marriages showed a premarital cohabitation.

- If the birth of the first child occurred before entering into marriage, the "child born before marriage" variable is 1 , otherwise it is 0 . Only children from the first marriage are taken into account.

- "Birth of first child" is a time-varying variable that is 0 up to the time the first marital child is born, and 1 afterwards.

- "Age at start of partnership" is measured in years for both partners. 
- "Education" of the respondents and their spouses is measured by the number of years that it usually takes to obtain a qualification. Educational years are categorized as follows: still attending school: 8 years; dropped out of primary/secondary school: 8 years; primary/secondary school: 9 years; middle school/ classical secondary school completion: 10 years; trade school completion: 12 years; high school completion (Abitur): 13 years. For the respondent, we know the highest qualification completed at the time of interview. For the spouse we know the educational level at the beginning of the partnership.

- An educational difference of the marriage partners is covered by a dummy variable. If the woman indicates at least two years of education more than her husband, then the variable takes the value 1 .

- Education of the respondent's father is measured by a dummy variable that is assigned a 1 if the "father completed high school" (Abitur). A father with Abitur tends to be indicative of a higher social origin.

- If the respondent has "no siblings", the variable takes on the value 1 , otherwise it is 0 .

- The parental family type is captured by three dummy variables: living up to the age of 15 predominantly with "no parents," "one parent," or "divorced parents." The reference category is those who still lived with both parents at age 15 .

\subsection{Estimation and Testing Procedures}

For estimation purposes covariables $x_{j}(j=1, \ldots, q)$ are generally included in the respective hazard rate model with an exponential link function through selected process parameters. In the sickle model the parameters $c$ and $\lambda$ are usually defined as follows (e.g., Diekmann and Engelhardt 1999):

$$
c=b_{c_{0}} \prod_{j=1}^{q} b_{c_{j}}^{x_{j}}, \lambda=b_{\lambda_{0}},
$$

where $b_{c 0}$ and $b_{\lambda 0}$ represent coefficients to be estimated and $b_{c j}$ the coefficient of the $j$ th covariable, which enters the model through the process parameter $c$. Employing the same notational logic, we follow the recommendation of Brüderl and Diekmann (1995) and define for the generalized log-logistic model accordingly: 


$$
\alpha=b_{\alpha_{0}}, \beta=b_{\beta_{0}} \prod_{j=1}^{q} b_{\beta_{j}}^{x_{j}}, \gamma=b_{\gamma_{0}} .
$$

Thus, covariables are included in the model through the process parameter $\beta$. Independently of whether we view the sickle model or the generalized log-logistic model, the estimated coefficients of the covariables indicate the "relative risks". The proportional rate effect of the $j$ th covariate is given by $100\left(b_{c j}-1\right) \%$.

If the vector of covariates $x_{i}$ and the marriage duration $t_{i}$ are known for each individual $i$, then the $b$-coefficients can be estimated (taking censored observations into account) consistently and (asymptotically) normally distributed by means of he maximum-likelihood method, which enables one to employ inference-statistical testing methods (cf., eg., Blossfeld, Hamerle and Mayer 1989). The log-likelihood function

$$
\ln L(b ; t, x)=\sum_{i=1}^{N}\left(d_{i} \ln h\left(t_{i} \mid x_{i}, b\right)-\int_{0}^{t_{i}} h\left(v \mid x_{i}, b\right) d v\right),
$$

which depends on the hazard function $h(\cdot)$ selected, is maximized. Here, $N$ defines the number of cases and $d_{i}$ is a binary variable which takes on the value 1 for uncensored observations. We obtain the maximum likelihood estimations using Rohwer's program TDA, though we used the technique of episode-splitting for the time-varying covariates (cf., e.g., Blossfeld and Rohwer 1995).

To evaluate the degree of improvement in the estimation ensuing from the addition of further parameters or covariates the Likelihood Ratio test is usually used. One compares thereby the maximized likelihood of the interesting (or unrestricted) model, $L_{U}$, with the maximized likelihood of the reference (restricted) model, $L_{R}$. The Likelihood Ratio test statistic $L R=2\left(\ln L_{U}-\ln L_{R}\right)$ is asymptotically $\chi^{2}$-distributed with the difference in parameters or covariates of the models under consideration as degrees of freedom. If $L R$ exceeds the relevant critical parameter, then the restrictions can be rejected (e.g., Brüderl 2000). Roughly speaking, the interesting model turns out to offer a significant improvement in the estimation.

In addition, the likelihood ratio statistic offers the basis for a comparison of nested models (e.g., the sickle model and the generalized log-logistic model) using the Bayesian Information Criterion BIC (Raftery 1995). One calculates for each model considered:

$$
-B I C=L R-\ln (N) z,
$$

where $z$ indicates the number of additional parameters in comparison to the selected reference model (e.g., exponential distribution). Usually, one considers the model with 
the highest $-B I C$ value to be the relatively "best" model. In the context of a selection to be undertaken on the basis of statistical criteria, this model would be selected.

\subsection{Results and Model Selection}

We compare now the sickle model and the generalized log-logistic model on this basis. We first consider the results of an estimation of both models without taking covariates into account (TABLE 2). The estimation results from the sickle model indicate that the maximum divorce risk occurs at approximately seven years $(\lambda=7.223)$, while the generalized log-logistic model fixes this point at somewhat over five years. ${ }^{15}$ In contrast to the sickle model, the generalized log-logistic model reproduces approximately the results of the non-parametric estimation (life table analysis and Kaplan Meier estimations), according to which the highest risk of divorce occurs at 4-5 years (Engelhardt 2000).

[Table 2 about here]

In addition to the estimated values for the process parameters, TABLE 2 also includes information about the test statistics and goodness-of-fit measures we have discussed. The exponential distribution without covariates serves in all cases as the reference model. ${ }^{16}$ If one uses the Bayesian Information Criterion BIC for model selection, then the generalized log-logistic model is to be preferred to the sickle model, judging from the estimated results for the scenario without covariates.

[Table 3 about here]

However, if one includes the covariates in the analysis, this results in the opposite conclusion, as can be seen in TABLE 3. Regardless of whether one carries out the comparison with the exponential model with or without Kovariablen, the sickle model presents itself as the "better" model due to the higher $-B I C$ value for the analysis of the

${ }^{15}$ According to Brüderl and Diekmann (1995) the timing of the maximal divorce risk arises in the case of the generalized log-logistic model as a result of $t_{m}=(1 / \gamma)(\alpha-1)^{(1 / \alpha)}$. When using the estimated results one arrives at $t_{m}=5.404$.

${ }^{16}$ The estimated value of the constant hazard rate in the exponential model is 0.008 . In comparison to this basic model with constant rate, the relative likelihood improvement achieved with the estimation from the Sickle model as well as from the generalized log-logistic model is small. When using McFaddens Pseudo$R^{2}$ (e.g., Brüderl 2000) as a conservative measure for the relative likelihood improvement one gets the value 0.01 for both models. If one considers in addition the covariates mentioned, this results in a Pseudo$R^{2}$ of about 0.06 for both models. 
divorce data under consideration. So if one chooses among parametric hazard rate models on the basis of statistical criteria, then the inclusion (or exclusion) of certain covariates obviously plays a substantial role in the decision-making process.

Sociologists are particularly interested now in the effects of covariates. One could take a pragmatic point of view and point out that the effects of covariates are practically identical in both models. From this perspective the question of model selection appears irrelevant in the case that the list of the covariates is fixed. However, this line of argumentation is not entirely convincing. The covariates can be sooner compatible with a certain type of the propagation than with another type. The covariates from TABLE 3 refer to individual and couple-specific characteristics, not, however, to relations with further persons and their characteristics. In this application interactions with unmarried or already divorced actors should therefore not be crucial components of the propagation process, which is determined by the choice of estimate model. Given the list of covariates, the assumption of a contact-independent propagation process seems appropriate for this example.

Furthermore, covariate effects are frequently interpreted with the help of theories that refer more or less explicitly to the individual decision behavior concerning the feature under consideration (e.g., divorce). For reasons of consistency, this theoretically founded micro-interpretation of covariate effects should not collide with the macroprocess, which is specified by the choice of parametric event model. Even if the covariates are fixed, for an adequate interpretation of their effects a process model has to be estimated that is compatible with the initial theoretical considerations.

Therefore, it seems useful to consider the process-theoretical implications discussed above when choosing the model. It is well known that the generalized log-logistic model illustrates a generalized infection process which is reduced to a pure infection scenario (log-logistic model) given a certain parameter constellation $(k=\gamma / \beta=1)$. It thus offers a test for whether the application under consideration can be interpreted as being a case of social infection. The estimated values of the parameters $\beta$ and $\gamma$ for the scenario without covariates in TABLE 2 then exclude an infection-theoretical interpretation of the divorce data $(\mathrm{k} \approx 15)$.

Instead, these values indicate, as expected, that the divorce risk depends less on interactions with third persons than on the adoption function, which is substantially determined by individual characteristics of the spouses and intra-couple processes. Moreover, if one considers the estimated results from TABLE 3, then most covariates exhibit significant and more or less strong effects on the risk of divorce. In all models this risk increases in comparison to the reference category (see section 4.1) if the interviewed person grew up in a broken home, has a father with Abitur, or grew up as an 
only child. The risk of divorce is also increased for couples without religious affiliation, of mixed denomination, or with different nationalities. ${ }^{17}$ Furthermore, the risk of divorce increases for younger marriage cohorts. In contrast, the affiliation of both husband and wife to the Catholic Church and the birth of the first conjugal child, for instance, decrease the risk.

Overall, these results are in agreement with the basic intuition that divorces result mainly from characteristics of the couple and intra-couple processes. With increasing time spent together, one acquires additional information about positive and negative characteristics of one's partner, and conflicts, boredom, or indifference can come about, factors that manifest themselves in an increasing inclination on the part of at least one of the partners to separate. These considerations are in keeping with existing theories of divorce research which form the basis of interpretations of the covariate effects. For example, according to Becker's economic theory of divorce (1991) it is the information about one's spouse acquired in the course of time which increases the likelihood of divorce. A general sense of disillusionment about one's spouse can arise as a result of additional experiences, or a mismatch can be diagnosed, so that an end to the relationship ultimately appears as the lesser evil.

In the analysis of divorce data, the effects of covariates are frequently interpreted in the sense of Becker's family economics. From a process-theoretical perspective such an interpretation is sooner compatible with the assumption of a contact-independent propagation. Accordingly, if one considers the event "divorce", then it makes sense to use the sickle model. If the underlying propagation logic is unclear in some other application with bell-shaped rate process, then such doubts might well be eliminated by the estimation of the generalized log-logistic model. Indeed, according to the model for connecting diffusion research and event analysis presented here, process-theoretical considerations facilitate the choice among competitive hazard rate models if statistical criteria do not offer us a clear basis for decision.

\footnotetext{
${ }^{17}$ The effects of the covariates need not necessarily be interpreted as causal effects. For example, the divorce risk as portrayed in TABLE 3 increases with pre-marital cohabitation. A more exact analysis of the connection between cohabitation and divorce indicates, however, that this positive relationship is due to a selection effect (Brüderl, Diekmann and Engelhardt 1997).
} 


\section{References}

Anderson, Roy M., and Robert M. May, 1991: Infectious Diseases of Humans: Dynamics and Control. Oxford: Oxford University Press.

Bailey, Norman T., 1975: The Mathematical Theory of Infectious Diseases and its Applications. London: Griffin.

Becker, Gary S., 1991: A Treatise on the Family, 2nd enlarged ed. Cambridge: Harvard University Press.

Blossfeld, Hans-Peter, Alfred Hamerle, and Karl Ulrich Mayer, 1989: Event History Analysis. Hillsdale, N.J.: Lawrence Erlbaum.

Blossfeld, Hans-Peter, and Götz Rohwer, 1995: Techniques of Event History Modeling: New Approaches to Causal Analysis. Hillsdale, N.J.: Lawrence Erlbaum.

Braun, Norman, 1998: Ansteckungstheoretische Begründungen für das Sichel-Modell und das generalisierte log-logistische Modell. Unpublished manuscript, Institute for Sociology, University of Berne.

Brüderl, Josef, 2000: Multivariate statistische Verfahren in der Bevölkerungswissenschaft. In: Ulrich Mueller, Andreas Diekmann und Bernhard Nauck (Hg.): Handbuch der Bevölkerungswissenschaft. Berlin: Springer, 589-642.

Brüderl, Josef, and Andreas Diekmann, 1995: The Log-Logistic Rate Model: Two Generalizations with an Application to Demographic Data, Sociological Methods \& Research 24: 158-186.

Brüderl, Josef, Andreas Diekmann, and Henriette Engelhardt, 1999: Premarital Cohabitation and Marital Stability in Germany.

Coleman, James S., Elihu Katz, and Herbert Menzel, 1957: The Diffusion of an Innovation among Physicians, Sociometry 20: 253-269.

Courgeau, Daniel, and Éva Lelièvre, 1992. Event History Analysis in Demography. Oxford: Clarendon Press.

Diekmann, Andreas, 1989: Diffusion and Survival Models for the Process of Entry into Marriage, Journal of Mathematical Sociology 14: 31-44.

Diekmann, Andreas, 1990: Hazard Rate Models of Social Diffusion Processes. Unpublished manuscript, Institute for Sociology, University of Berne.

Diekmann, Andreas, 1992: The Log-logistic Distribution as a Model for Social Diffusion Processes, Journal of Scientific \& Industrial Research 51: 285-290.

Diekmann, Andreas, and Henriette Engelhardt, 1999: The Social Inheritance of Divorce: Effects of Parent's Family Type in Postwar Germany, American Socioplogical Review 64: 783-793.

Diekmann, Andreas, and Peter Mitter, 1983: The "Sickle Hypothesis": A TimeDependent Poisson Model with Applications to Deviant Behavior and Occupational Mobility, Journal of Mathematical Sociology 9: 85-101. 
Diekmann, Andreas, and Peter Mitter, 1984: Methoden zur Analyse von Zeitverläufen. Stuttgart: Teubner.

Diekmann, Andreas, and Peter Mitter, 1984a: A Comparison of the "Sickle Function" with Alternative Stochastic Models of Divorce Rates. Pp.123-153 in: Andreas Diekmann and Peter Mitter (eds.): Stochastic Modelling of Social Processes. Orlando: Academic Press.

Engelhardt, Henriette, 2000: Zur Dynamik von Ehescheidungen. Berlin: Duncker \& Humblot (forthcoming).

Hamblin, Robert L., R. Brooke Jacobsen, and Jerry L.L. Miller, 1973: A Mathematical Theory of Social Change. New York: Wiley.

Kramer, Michael S., 1988: Clinical Epidemiology and Biostatistics. Berlin, Heidelberg, New York: Springer.

Lancaster, Tony, 1996. Econometric Analysis of Duration Data. Cambridge: Cambridge University Press.

Mahajan, Vijay, and Robert A. Peterson, 1985: Models for Innovation Diffusion. Beverly Hills: Sage.

Morris, Martina, 1994: Epidemiology and Social Networks: Modeling Structured Diffusion. Pp. 26-52 in: Stanley Wasserman and Joseph Galaskiewicz (eds.): Advances in Social Network Analysis: Research in the Social and Behavioral Sciences. Thousand Oaks: Sage.

Raftery, Adrian E., 1995: Bayesian Model Selection in Social Research. Pp. 111-163 in: Peter V. Marsden (ed.): Sociological Methodology 1995. Oxford: Blackwell.

Rogers, Everett M., 1983: Diffusion of Innovations, 3rd ed. New York: Free Press.

Strang, David, 1991: Adding Social Structure to Diffusion Models: An Event History Framework, Sociological Methods \& Research 19: 324-353.

Yamaguchi, Kazuo, 1994: Some Accelerated Failure-Time Regression Models Derived From Diffusion Process Models: An Application to a Network Diffusion Analysis. Pp. 267-300 in: Peter V. Marsden (ed.): Sociological Methodology 1994. Oxford: Blackwell. 
TABLE 1: Special cases of the diffusion model*

Logistic model (spreading through infection):

$k=1$ as well as $c>0, \delta=0$ and $\varepsilon=1$,

so that $w(t)=F(t), r(t)=c$ and $h(t)=c F(t)$

Log-logistic model (spreading through infection):

$k=1$ as well as $c>0, \delta=0$ and $\varepsilon=0$,

so that $w(t)=F(t), r(t)=c / t$ and $h(t)=(c / t) F(t)$

Generalized log-logistic model (spreading through contacts):

$k=\gamma / \beta>0$ as well as $c=\alpha \beta / \gamma>0, \delta=0, \varepsilon=0$,

so that $w(t)=1-(1-F(t))^{\gamma / \beta}, r(t)=\alpha \beta / \gamma t$

and $h(t)=(\alpha \beta / \gamma t)\left(1-(1-F(t))^{\gamma / \beta}\right)$

Sickle model (contact-independent spreading):

$k \rightarrow \infty$ as well as $c>0, \delta=-1 / \lambda<0$ and $\varepsilon=2$,

so that $w(t)=1$ and $r(t)=c t e^{-t / \lambda}=h(t)$

Exponential model (contact-dependent spreading):

$k \rightarrow \infty$ as well as $c>0, \delta=0$ and $\varepsilon=1$,

so that $w(t)=1$ and $r(t)=c=h(t)$

* Further model descriptions can be found in textbooks on event history analysis (e.g., Blossfeld, Hamerle and Mayer 1989; Blossfeld and Rohwer 1995; Diekmann and Mitter 1984). 
TABLE 2: Maximum likelihood estimations of the divorce risk for two models without covariates

\begin{tabular}{lll}
\hline \hline $\begin{array}{l}\text { Parameter and } \\
\text { test statistics }\end{array}$ & $\begin{array}{l}\text { Sickle } \\
\text { model }\end{array}$ & $\begin{array}{l}\text { Generalized log- } \\
\text { logistic model }\end{array}$ \\
\hline$c$ & $0.005^{* * *}$ & - \\
$\lambda$ & $7.223^{* * *}$ & - \\
$\alpha$ & - & $1.928^{* * *}$ \\
$\beta$ & - & $0.012^{* * *}$ \\
$\gamma$ & - & $0.178^{* * *}$ \\
\hline- Log-likelihood & 4528.47 & 4523.42 \\
$L R$ & 90.90 & 101.00 \\
$d f$ & 1 & 2 \\
$-B I C$ & 82.16 & 83.52 \\
\hline \hline
\end{tabular}

Notes: *** significant for $\mathrm{p} \leq .001 . L R$ is the likelihood-ratio statistik with $d f$ degrees of freedom. $B I C$ is the Bayesian Information Criterion with the exponential model without covariates as reference model $($ Log-Likelihood $=-4573.92) . N=6247$. 
TABLE 3: Maximum likelihood estimations of the divorce risk for two models with covariates

\begin{tabular}{lll}
\hline \hline Covariates, parameters & Sickle & $\begin{array}{l}\text { Generalized log- } \\
\text { logistic model }\end{array}$ \\
and test statistics & model & $0.131^{* * *}$ \\
\hline Constant & $0.037^{* * *}$ & $1.792^{* * *}$ \\
Married 1961-70 & $1.744^{* * *}$ & $2.417^{* * *}$ \\
Married 1971-80 & $2.383^{* * *}$ & $2.686^{* * *}$ \\
Married 1981-88 & $2.742^{* * *}$ & $0.650^{* * *}$ \\
Catholic couple & $0.650^{* * *}$ & $2.281^{* * *}$ \\
Nonreligious couple & $2.276^{* * *}$ & $1.339^{* * *}$ \\
Mix of religions & $1.339^{* * *}$ & $1.780^{* * *}$ \\
Mix of nationalities & $1.802^{* * *}$ & $1.664^{* * *}$ \\
Cohabitation & $1.670^{* * *}$ & 0.995 \\
Child before marriage & 0.982 & $0.447^{* * *}$ \\
First child (time dependent) & $0.438^{* * *}$ & $0.920^{* * *}$ \\
Age at marriage & $0.920^{* * *}$ & 0.960 \\
Male: years of education & 0.961 & 0.952 \\
Wife: years of education & 0.952 & 0.957 \\
Wife has 2+ years more educ. & 0.956 & $1.352^{* *}$ \\
No siblings & $1.352^{* *}$ & $1.519^{* *}$ \\
Father: Abitur & $1.519^{* *}$ & $1.660^{* *}$ \\
No parents & $1.664^{* *}$ & 1.209 \\
One parent & 1.209 & $1.972^{* * * *}$ \\
Divorced parents & $1.973^{* * *}$ & - \\
\hline$c$ & par. & - \\
$\lambda$ & $9.906^{* * *}$ & $1.924^{* * * *}$ \\
$\alpha$ & - & par. \\
$\beta$ & - & $0.113^{* * * *}$ \\
$\gamma$ & - & 4308.96 \\
\hline L Log-likelihood & 4310.47 & 129.56 \\
$d f$ & 126.54 & 112.08 \\
$-B I C$ & 20 & \\
\hline \hline
\end{tabular}

Notes: ** significant for $\mathrm{p} \leq .01, * * *$ significant for $\mathrm{p} \leq .001$. Reported are the $b$-coefficients of the covariates, which determine the "par."-marked process parameters. $L R$ is the likelihood-ratio statistic with $d f$ degrees of freedom. BIC is the Bayesian Information Criterion with the exponential model without covariates as reference model $(\log -$ likelihood $=-4373.74)$. A comparison with the exponential model without covariates yields $L R(-B I C)=526.90(518.16)$ for the sickle model and $L R(-B I C)=529.92$ (512.44) for the generalized log-logistic model. $N=6247$; number of splits $=10791$. 